\title{
Respostas da pressão arterial de futebolistas adolescentes ao exercício em escada coordenativa*
}

\author{
Response of blood pressure of adolescents \\ soccer players to exercise on coordinate \\ ladder
}

João Batista da Silva Soares ${ }^{1}$ Lia Nara de Moraes Guazelli ${ }^{2}$ Renata Aparecida Elias Dantas ${ }^{3}$ Edísio Sobreira Gomes de Matos Filho ${ }^{4}$ Eduardo Silva Trindade ${ }^{5}$ Rafaela Barbosa de Sousa ${ }^{6}$ Sylvestre da Silva Alberto Júnior ${ }^{7}$ Emerson Pardono ${ }^{8}$ Márcio Rabelo Mota ${ }^{9}$

Recebido em: 21/09/2016.

Aprovado em: 20/01/2017.

1 Professor de Educação Física no ICPD-CESAPE, email: joaobatistassoares@gmail.com.

2 Professora do Curso de Educação Física do Universidade de Brasília, UnB, email: lianmg@ hotmail.com.

3 Professora do Curso de Educação Física do UniCEUB, email: profrenataelias@yahoo.com. br.

4 Pós Graduado em Fisiologia do Exercício, ICPD-CESAPE, email: edisiosobreira@hotmail. com.

5 Professor do Curso de Educação Física no ICPD-CESAPE, email: eduardotridi@hotmail.com.

6 Especialista em Fisiologia do Exercício, ICPD-CESAPE, email: joaobatistassoares@gmail. com.

7 Professor do Curso de Educação Física no ICPD-CESAPE, email: sylvestre@globo.com.

8 Professor da Universidade Federal de Sergipe, email: pardono@gmail.com.

9 Professor do Curso de Educação Fisica do UniCEUB, email: marciormota@gmail.com.

\section{Resumo}

O futebol figura entre as modalidades mais praticadas do mundo. As síndromes metabólicas, atualmente, são um tema de grande relevância no contexto do futebol, quando relacionadas aos problemas cardiovasculares. No futebol as capacidades físicas são importantes para suprir as necessidades do atleta. Foram analisadas as respostas da pressão arterial pré e imediatamente após exercício moderado e intenso em futebolistas adolescentes. O estudo foi realizado com 10 futebolistas $(\mathrm{n}=10)$ do gênero masculino de 14 a 16 anos, em 02 dias distintos e intervalo de descanso de 24 horas. Exercícios de aquecimento duraram de 10 minutos de forma aguda moderada e 05 minutos aguda intensa, avaliados dois a dois. Exercícios realizados na escada coordenativa. Os dados evidenciaram que houve alteração significativa na Pressão Arterial Sistólica (PAS) entre o pré e imediatamente após exercício. Os exercícios mostraram-se como boas opções de aquecimento na preparação física do futebol juvenil.

Palavras-chave: Futebol. Exercício Físico. Pressão Arterial.

\begin{abstract}
Soccer is among the most widely practiced forms of the world. The metabolic syndromes, currently appearing in a highly relevant issue in the context of football, when related to cardiovascular problems. In football the physical capabilities are important to meet the meeds atleta. The study analyzed the responses of pre arteral pressure and immediately after moderate and intense exercise in soccer players adolescents. The study consisted of ten male and female soccer players $(n=10)$ from 14 to 16 years old, performed on two distinct days and a 24-hour rest interval. Warm-up exercise lasted from 10 minutes to a moderate acute and 5 minutes to an acute, two-minute evaluation. Exercises carried out in the coordinative scale. Data showed that there was a significant change in systolic blood pressure (SBP) between pre and immediately after exercise. The exercises were observed as good heating options in the physical preparation of youth soccer.
\end{abstract}

Keywords: Soccer. Physical exercise. Blood pressure. 


\section{Introdução}

O futebol figura entre as modalidades mais praticadas do mundo, independentemente de classe social, sexo, etnia, idade e religião (SALLES, 2012).

Os atletas estão expostos a síndromes que atingem o coração. Tais síndromes se encontram em grande relevância no contexto do futebol, já que os motivos que provocam essas alterações cardiovasculares e suas respostas fisiológicas, ainda, não foram esclarecidas plenamente (CASTANHEIRA; PEREIRA; CONDE, 2007).

A Hipertensão é um mal que ocorre com grande incidência em todas as populações e não é diferente na esfera esportiva (SCHRAMMEL, 2015). A Pressão Alta se traduz na força que o fluxo de sangue exerce nas paredes internas dos vasos sanguíneos e são vários fatores que exercem essa pressão (SBC, 2010). Acontece a pré-hipertensão quando a Pressão Arterial Sistólica (PAS) está na faixa de 120 - 139 mmHg ou a Pressão Arterial Diastólica equivalente a 80 89 mmHg (POWERS; HOWLEY, 2014, p. 194).

A Organização Mundial de Saúde (OMS), em seu relatório anual que fala sobre estatísticas sanitárias, afirma que de cada três adultos, um sofre de hipertensão, fator que causa a metade das mortes por derrame e doenças do coração no mundo (SBC, 2010).

As causas da Hipertensão são diversas e muitos casos iniciam na infância (COSTA et al, 2012). O fator ge-

nético, o estilo de vida e a alimentação inadequada são as principais causas de riscos das doenças cardiovasculares e que contribuem para o surgimento de outras patologias que atingem a população mundial (GUEDES, 2010).

O meio mais barato e saudável, para redução da hipertensão, é a prática de exercícios físicos, além de ajudar na reabilitação cardíaca, melhora a resistência, a força muscular e a qualidade de vida, por meio de atitudes simples como: caminhar, pedalar, correr ou nadar (BAGANHA et al., 2014).

A inatividade física é um fator de risco primário para as doenças coronarianas e outras patologias associadas ao estilo de vida e à alimentação inadequada, como diabete, obesidade e hipertensão (SAMPAIO et al, 2013). Pessoas que praticam algum tipo de exercício físico tendem a melhorar o condicionamento cardiorrespiratório, quando comparadas com as pessoas inativas e sedentárias, as taxas de mortalidades são menores (DUTRA et al., 2008).

Os futebolistas são submetidos a exercícios moderados e intensos a todo momento e a todo instante ocor- exercícios, frequentemente impostas ao coração do jogador, são os exercícios dinâmicos e isométricos (DUTRA, 2013). Quando esse contexto do futebol é relacionado aos problemas cardiovasculares, constitui um tema de grande interesse, até pela falta de parâmetros adequados entre as respostas cardiovasculares fisiológicas e as de causa patológicas na área do treinamento esportivo; principalmente quando ocorrem casos em que futebolistas morrem de ataque cardíaco durante o jogo (MENESES et al., 2015).

De acordo com Falk (2010), a partida de futebol é o cenário ideal para os futebolistas exibirem o preparo físico, o controle emocional, a força, a técnica apurada, realizar movimentos cíclicos e acíclicos coordenados, com velocidade, agilidade e potência. No futebol a importância das duas vias metabólicas aeróbia e anaeróbia são evidentes, as capacidades físicas devem ser muito bem desenvolvidas para que as duas vias possam suprir o futebolista no momento de resistência ou em um momento de velocidade no jogo. A prevalência do fator aeróbio, na maior parte do jogo, não tira o poder e decisão do fator anaeróbio que prevalece nos momentos decisivos.

O objetivo do presente estudo foi analisar as respostas da pressão arterial pré e, imediatamente, após exercício moderado e intenso em futebolistas de 14 a 16 anos de idade.

\section{Materiais e Métodos} rem respostas morfofisiológicas e funcionais; as cargas de

\subsection{Aspectos Éticos}

O estudo foi realizado como pesquisa exploratória, desenvolvida com base no projeto de pesquisa enviado ao Comitê de Ética da Faculdade de Ciências da Educação e Saúde do Centro Universitário de Brasília - UniCEUB e aprovado sob o CAAE 48235715.9.0000.0023.

\subsection{Amostra}

Participaram da amostra 10 futebolistas $(n=10)$ do gênero masculino, com faixa etária de 14 a 16 anos de idade da escola de futebol Colorado Esporte Clube Paranoá, com média de idade de $15,10 \pm 0,88$ e média de massa corporal $61,15 \pm 4,72$, estatura média de 174,10 $\pm 5,28$. Tendo a média de IMC $\left(\mathrm{kg} / \mathrm{m}^{2}\right)$ de $20,20 \pm 0,92$. A pesquisa foi autorizada e oficializada pela Secretaria Executiva do Clube, sendo iniciada após a autorização expressa no Termo de Consentimento Livre e Esclarecido (TCLE) mediante assinatura dos participantes do estudo e respectivamente seu responsável. 
A seleção dos participantes atendeu aos seguintes critérios de inclusão: estar matriculado na escola de futebol do Colorado Esporte Clube Paranoá, concordar com as avaliações antropométricas e de pressão arterial durante o estudo e não possuir histórico de doenças cardiovasculares ou osteomioarticulares de qualquer segmento dos membros inferiores, que impeçam a realização dos exercícios propostos neste estudo. Os participantes foram, também, informados que os dados coletados serviram de análise para estudo acadêmico.

\subsection{Materiais}

Para a coleta de dados, foram utilizados os materiais apresentados no Quadro 1.

Quadro 1 - Materiais utilizados na coleta de dados.

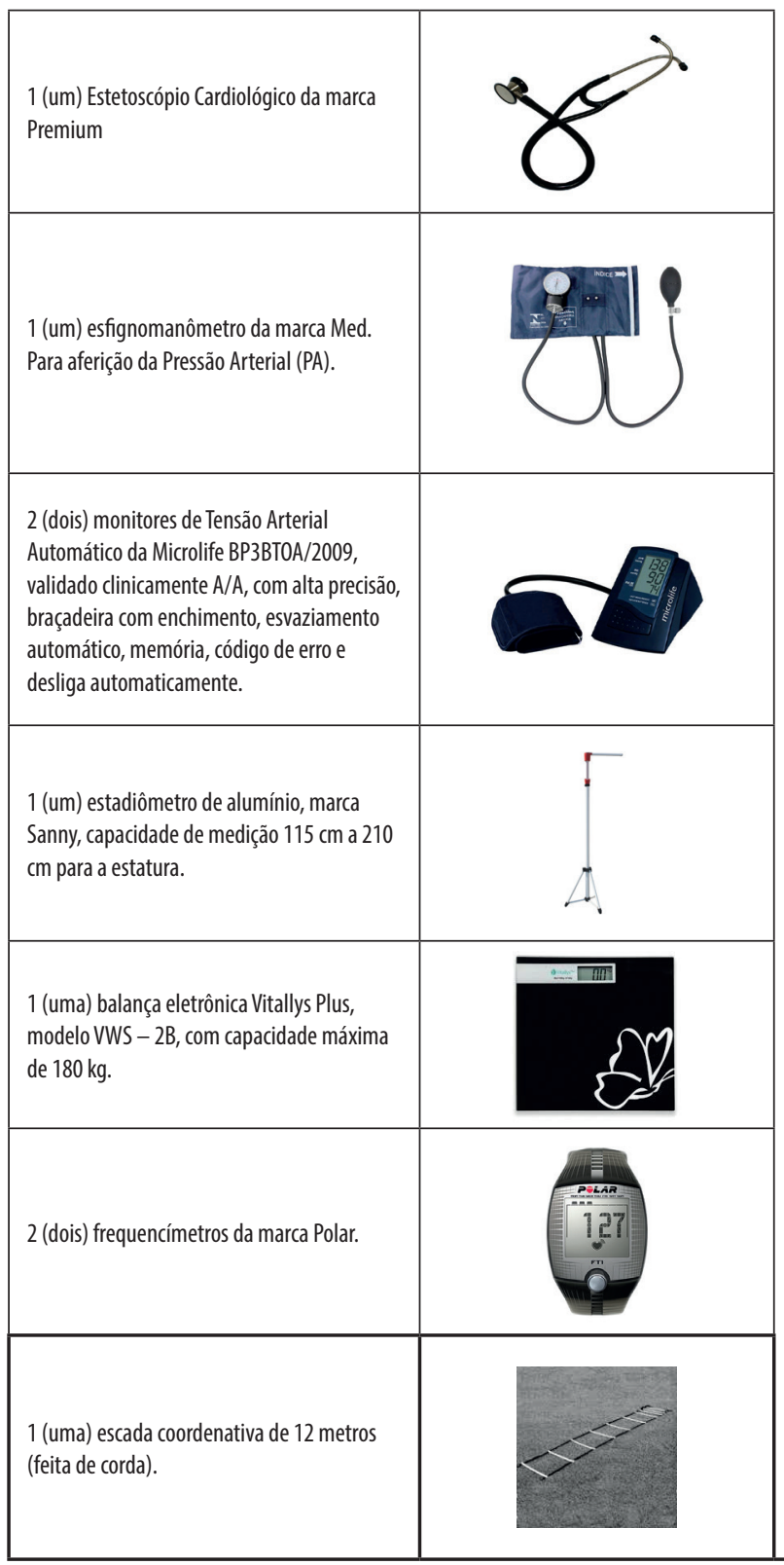

Fonte: Elaborada pelos autores do trabalho.

\subsection{Métodos}

A amostra foi composta por 10 futebolistas. O estudo foi realizado em 02 dias distintos, intervalo aproximadamente 24 horas de descanso. Os exercícios de aquecimento tiveram duração de 10 minutos, de forma aguda, moderada de 70 a $80 \%$ da frequência cardíaca máxima e 05 minutos de forma aguda intensa de 80 a 90\% da frequência cardíaca máxima, foram realizados os mesmos exercícios para as duas intensidades. Os avaliados fizeram os exercícios em duplas (Figura 1). Os exercícios foram realizados na escada coordenativa pelos atletas, que realizaram movimentos frontais, posteriores, laterais e movimentos pliométricos de forma continua. Na percepção de esforço, foi utilizado o frequencímetro, no controle da frequência cardíaca.

Figura 1 - Exercícios de aquecimento sendo realizados em pares para posterior avaliação.

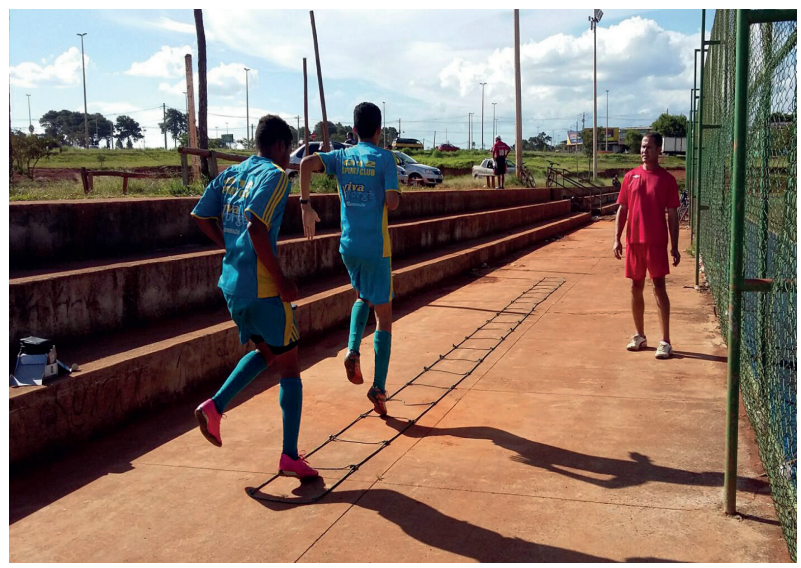

Fonte: Elaborada pelos autores do trabalho.

Calculou-se o índice de massa corporal (IMC) e os valores de ponto de corte para definir peso normal, sobrepeso e obesidade. Para aferir o peso corporal dos futebolistas, foi utilizada uma balança eletrônica e eles foram pesados com o mínimo de roupa possível, conforme padrões protocolados.

\subsection{Protocolos e procedimentos}

\subsubsection{Medidas Antropométricas (Peso, Altura, IMC)}

Os futebolistas foram pesados com o mínimo de roupa possível, os pés, ligeiramente, separados na balança e descalços. Na mensuração da estatura, eles ficaram com os pés juntos e descalços e com a postura ereta, ver Figura 2. 
Figura 2 - Momento de aferição da estatura.

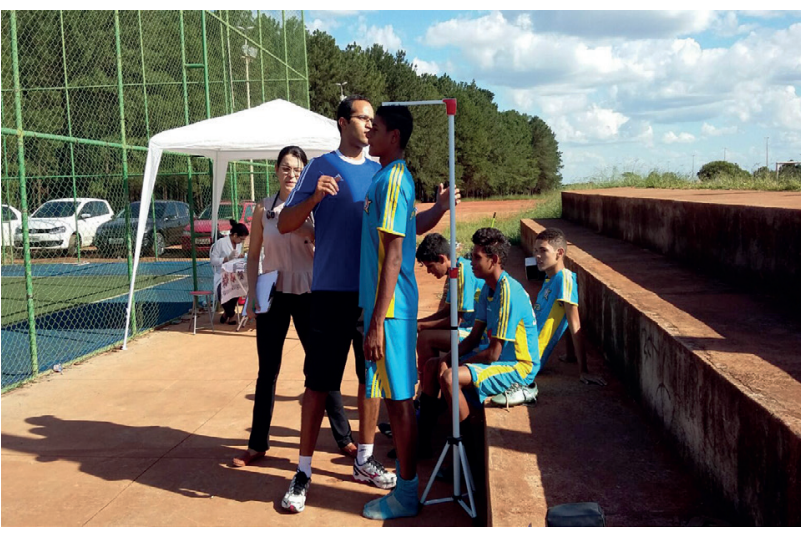

Fonte: Elaborada pelos autores do trabalho.

\subsubsection{Avaliação da Pressão Arterial Sistólica e Di- astólica}

A aferição da Pressão Arterial Sistólica (PAS) e Pressão Arterial Diastólica (PAD) pré-exercício foram realizadas no medidor automático de pressão arterial, em uma tenda armada próxima ao campo de treino. Os futebolistas ficaram sentados durante 5 minutos, descansados, relaxados e em total silêncio, o braço na altura adequada (ponto médio do externo) e ao tempo de deflação do manguito. A aferição da Pressão Arterial imediatamente após o exercício foi realizada no campo pelo modo auscultatório utilizando um estetoscópio cardiológico, ver Figura 3.

Figura 3 - Mesa preparada para aferição da pressão arterial.

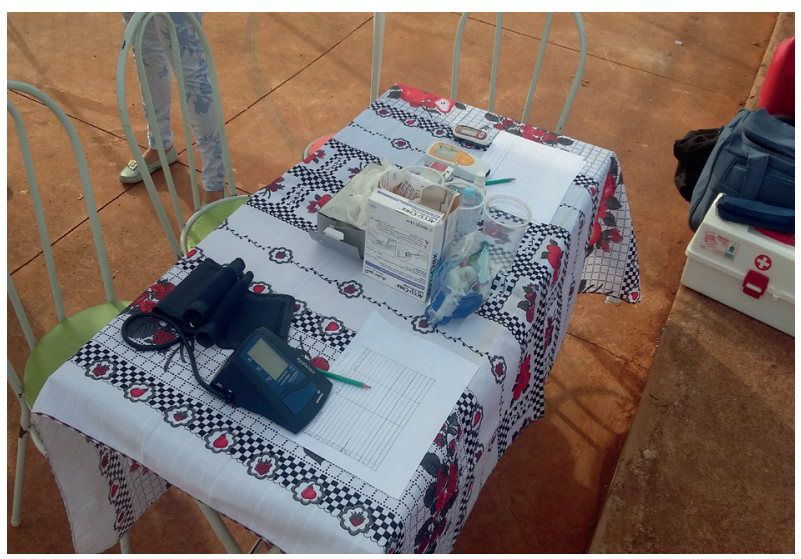

Fonte: Elaborada pelos autores do trabalho.

\subsection{Análise Estatística}

Os dados foram expressos em média \pm desvio padrão. A normalidade dos dados foi verificada por meio do teste de Shapiro-Wilk. O comportamento da PAS e da PAD antes e após os exercícios moderado e intenso foram comparados por meio da análise de variância ANOVA fatorial de medidas repetidas $2 \times 2$ (exercício versus momento), com tratamento de Bonferroni. Todas as análises foram realizadas no software estatístico SPSS versão 21.0. Adotou-se como nível de significância $\mathrm{p}<0,05$.

\section{Resultados}

Os dados referentes à caracterização amostral estão expostos na Tabela 1.

Tabela 1 - Variáveis de caracterização amostral expressas em média e desvio padrão.

\begin{tabular}{lc}
\hline Idade (anos) & $15,10 \pm 0,88$ \\
Massa Corporal (kg) & $61,15 \pm 4,72$ \\
Estatura (m) & $174,10 \pm 5,28$ \\
IMC $\left(\mathrm{kg} / \mathrm{m}^{2}\right)$ & $20,20 \pm 0,92$
\end{tabular}

Fonte: Elaborada pelos autores do trabalho.

A PAS apresentou aumento significativo entre o momento pré e o momento pós-exercício moderado $(\mathrm{p}<$ $0,001)$ e entre o momento pré e o momento pós-exercício intenso ( $\mathrm{p}<0,001)$. Não houve diferença significativa entre as duas intensidades de exercício no momento pré ( $\mathrm{p}$ $=1,000)$, nem no momento pós $(\mathrm{p}=0,093)$. A PAD não apresentou alteração significativa em nenhum momento nas duas intensidades de exercício ( $\mathrm{p}>0,05)$, conforme exporto na Tabela 2.

Tabela 2 - Resposta da PAS e da PAD antes e após as duas intensidades de exercício, expressa em média e desvio padrão, e significância.

\begin{tabular}{lccc}
\hline PAS (mmHg) & Pré exercício & Pós Exercício & p \\
Exercício Moderado & $118,20 \pm 12,27$ & $147,90 \pm 17,15$ & $<0,001$ \\
Exerć́cio Intenso & $122,60 \pm 11,67$ & $167,80 \pm 15,57$ & $<0,001$ \\
& & & \\
PAD (mmHg) & & & \\
Exerć́cio Moderado & $72,50 \pm 9,12$ & $85,60 \pm 10,39$ & 0,095 \\
Exercício Intenso & $81,20 \pm 8,99$ & $83,30 \pm 12,51$ & 1,000 \\
\hline
\end{tabular}

Fonte: Elaborada pelos autores do trabalho. 


\section{Discussão}

O presente estudo demonstrou, por meio de duas sessões de exercícios dinâmicos com intensidades moderada e alta, de forma aguda, as respostas da Pressão Arterial no contexto do futebol. O resultado da Pressão Arterial Sistólica (PAS) apresentou aumento significativo entre o momento pré e o momento pós exercício moderado $(\mathrm{p}<0,001)$ e entre o momento pré e o momento pós-exercício intenso $(\mathrm{p}<0,001)$.

No estudo de SILVA et al. (1997), foram analisadas as respostas cardiorrespiratórias e metabólicas de 16 jogadores de futebol profissional, sexo masculino, com média de idade de $24,2 \pm 3,6$ anos, peso de 75,0 $\pm 8,0 \mathrm{~kg}$ e estatura de $179 \pm 5 \mathrm{~cm}$. Nesse trabalho, os pesquisadores efetuaram o treinamento físico específico (TFE), e evidenciaram que houve diferença na PAS pós comparada com a pré e com diferença significativa de $\mathrm{P}<0,05$, desta maneira, essas informações vão de encontro com o presente estudo devido à pressão arterial ter se elevado, com diferença significativa de $\mathrm{p}<0,05$.

Fisiologicamente, em indivíduos normotensos, o aumento da Pressão Arterial Sistólica (PAS), durante o exercício dinâmico, ocorre por que aumenta o volume do sangue circulante, como consequência a vasoconstrição de áreas relacionadas aos sistemas hepático, esplênico e renal, gerando o aumento do retorno do volume venoso, sistólico e aumento da pressão dentro do vaso (CONCEIÇÃO et al., 2006). O efeito contrário acontece na Pressão Arterial Diastólica (PAD), durante a vasodilatação periférica, que diminui a pressão dentro do vaso e acomoda todo retorno do volume sistólico (TERRA et al., 2008). Quando ocorre o desajuste desses mecanismos, acontece o aumento excessivo da Pressão Arterial Sistólica (PAS) e/ou da Pressão Arterial Diastólica (PAD) durante o exercício, caracterizando resposta hipertensiva (CARREIRA, 2008). O indivíduo só é considerado hipertensivo se a PAS for maior que $220 \mathrm{mmHg}$ no pico do esforço e/ou a pressão arterial diastólica tiver variação ao pico do esforço maior que $15 \mathrm{mmHg}$ (SBC, 2010).

Não houve diferença significativa entre as duas intensidades de exercício no momento pré $(\mathrm{p}=1,000)$, nem no momento pós $(p=0,093)$. A PAD não apresentou alteração significativa em nenhum momento nas duas intensidades de exercício ( $p>0,05)$, conforme exporto na Tabela 2.

No estudo de MOTA et al. (2007), em que foi analisado o delta de variação da PAS e PAD pós-exercício aeróbio com duração de 20 minutos em sujeitos normotensos, a amostra então composta por 8 voluntários (6 homens e 2 mulheres) fisicamente ativos, foi submetida a duas sessões de exercícios físicos aeróbios em uma esteira ergométrica, em dias distintos e em um mesmo período do dia, e com intervalos mínimos 48 horas entre as sessões. Na primeira sessão, foi realizado exercício aeróbio em esteira ergométrica; e na segunda sessão foi dia controle sem realização de exercício. Apesar dos estudos serem realizados sobre o exercício aeróbio, terem a amostragem parecida e analisar a variação da Pressão Arterial Sistólica (PAS) e da Pressão Arterial Diastólica (PAD), possuem características diferentes, ambos comprovaram que houve diferença significativa na PAS pós comparada com a pré $(\mathrm{p}<0,05)$.

\section{Considerações Finais}

Os resultados do presente estudo mostraram que houve alteração significativa na Pressão Arterial Sistólica (PAS) entre o pré e pós exercício. O estudo evidenciou na prática que o aquecimento pode ser realizado nas duas formas de intensidades de exercícios, com duração de 10 minutos na forma moderada de 70 a $80 \%$ da frequência cardíaca máxima e 05 minutos na forma intensa de 80 a $90 \%$ da frequência cardíaca máxima. Apesar de o estudo não ter mostrado diferença significativa entre as duas intensidades, os exercícios moderados e intensos mostraram-se boas opções no aquecimento para a preparação física no futebol para adolescentes.

\section{Referências}

BAGANHA R. J. et al. Hipertensão arterial sistêmica e exercício físico: adaptações e mecanismos hipotensores associados. Revista Brasileira de Prescrição e Fisiologia do Exercício, São Luis, v. 8, n. 47, p. 499-506, dez. 2014.

CARREIRA, M. A. M. Q. Contribuição do Teste de Esforço na Orientação Terapêutica da Hipertensão Arterial. In: CONGRESSO NACIONAL DERC, 15., 2008. Anais... Rio de Janeiro: DERC, 2008.

CASTANHEIRA J.; PEREIRA, T.; CONDE, J. Adaptações cardíacas em atletas: Estudo comparativo. In: CONGRESSO VIRTUAL DE CARDIOLOGIA,5., 2007. Anais... Coimbra: CVC, 2007.

CONCEIÇÃO, T. V. et al. Valores de pressão arterial e 
suas associações com fatores de risco cardiovasculares em servidores da Universidade de Brasília. Arquivos Brasileiros de Cardiologia, São Paulo, v. 86, n. 1, p. 26-31, jan. 2006. doi: 10.1590/S0066-782X2006000100005

COSTA, J. V. et al. Análise de fatores de risco para hipertensão arterial em adolescentes escolares. Revista Latino-Americana de Enfermagem, Ribeirão Preto, v. 20, n. 2, p. 289-295, Apr./May 2012. doi: 10.1590/S010411692012000200011

DUTRA, M. T. et al. Estudo Comparativo do Efeito Hipotensor de Diferentes Modalidades Aeróbias em Mulheres Normotensas. Revista da Educação Física/UEM, Maringá, v. 19, n. 4, p. 549-556, out./dez. 2008. doi: 10.4025/ reveducfis.v19i4.5138

DUTRA, M. T. et al. Hipotensão Pós-Exercício Resistido: Uma Revisão da Literatura. Revista da Educação Física/ UEM, Maringá, v. 24, n. 1, p. 145-157, jan./mar. 2013. doi: 10.4025/reveducfis.v24i1.16927

FALK, P. R. A.; PEREIRA, D. P. Futebol: gestão e treinamento. São Paulo: Ícone, 2010.

GUEDES, N. G.; LOPES, M.V.O. Exercício físico em portadores de hipertensão arterial: uma análise conceitual. Revista Gaúcha de Enfermagem, Porto Alegre, v. 31 , n. 2, p. 367, jun. 2010. doi: 10.1590/S198314472010000200023

MENESES, D. B. et al. Morte Súbita em Jogadores de Futebol Relacionado à Cardiomiopatia Hipertrófica. Núcleo Interdisciplinar de Pesquisa. 2015. Disponível em: <http://nippromove.hospedagemdesites. ws/anais_simposio/arquivos_up/documentos/artigos/ f48bbf9629904786eb5dddb828d70d80.pdf>. Acesso em: 05 abr. 2017.
MOTA, M. R. et al. Efeito do Exercício Aeróbio sobre a Hipotensão Pós-Exercício. Coleção Pesquisa em Educação Física, São Paulo, v. 6, n. 2, p. 311-316, set. 2007.

POWERS, S. K.; HOWLEY, E. T. Fisiologia do Exercício: Teoria e Aplicação ao Condicionamento e ao Desempenho. 8. ed. São Paulo: Manole, 2014.

SALLES, J. G. C. Escola de futebol: criação, seleção de atividades, planejamento, organização e controle. Várzea Paulista: Fontoura, 2012.

SAMPAIO, W. B. et al. Estudo Comparativo da Pressão Arterial Pós-Exercício entre o Karate de Contato e o Karate Tradicional. Revista Brasileira de Ciência e Movimento, Brasília, v. 21, n. 4, p. 13-20, out./dez. 2013. doi: 10.18511/0103-1716/rbcm.v21n4p13-20

SOCIEDADE BRASILEIRA DE CARDIOLOGIA. VI Diretrizes Brasileiras de Hipertensão Arterial. Arquivos Brasileiros de Cardiologia, São Paulo, v. 95, n. 1 Suppl. 1, p. 1-51, dez. 2010. doi: 10.1590/S0066782X2010001700001.

SCHRAMMEL. A. C. Intensidade do Exercício Físico e o Risco Cardíaco em Praticantes de Futebol de Campo amador. 49f. Trabalho de Conclusão de Curso (Graduação) - Educação Física na UNIJUI, Santa Rosa, 2015.

SILVA, P. R. S. et al. Efeitos do treinamento físico específico nas respostas cardiorrespiratórias e metabólicas em repouso e no exercício máximo em jogadores de futebol profissional. Acta Fisiátrica, São Paulo, v. 4, n. 2, p. 5964, ago. 1997.

TERRA, D. F. et al. Redução da pressão arterial e do duplo produto de repouso após treinamento resistido em idosas hipertensas. Arquivos Brasileiros de Cardiologia, São Paulo, v. 91, n. 5, p. 299-305, nov. 2008. doi: 10.1590/ S0066-782X2008001700003 